\title{
Is there still a place for open surgery in the treatment strategy of rectal cancer?
}

\author{
Andrzej Kawiński, Łukasz Dziki, Radzisław Trzciński, Adam Dziki, Michał Mik \\ Department of General and Colorectal Surgery, Medical University of Lodz, Lodz, Poland
}

Gastroenterology Rev 2018; 13 (4): 289-292

DOI: https://doi.org/10.5114/pg.2018.79807

Key words: rectal cancer, laparoscopy, open surgery, conversion rate.

Address for correspondence: Michał Mik MD, PhD, Ass. Prof., Department of General and Colorectal Surgery, Medical University of Lodz, Plac Hallera 1, 90-647 Lodz, Poland, phone: +48 4263930 74, fax: +48 4263930 76, e-mail: m.mik@wp.pl

\begin{abstract}
Over the last 30 years laparoscopic surgery of rectal cancer has been progressively gaining popularity due to expanding experience and the development of technical skills according to the surgeons' learning curves. The report constitutes a review of recently published articles concerning surgical techniques in colon cancer, with special emphasis on rectal cancer treatment. We tried to address the question of whether, in view of further development and broader use of laparoscopic surgery, there still is a place for open surgery in the treatment strategy of rectal cancer.
\end{abstract}

\section{Introduction}

Since the development of the total mesorectum excision (TME) technique by Heald and Ryall in the 1980s the efficacy of a surgical approach in rectal cancer treatment has systematically increased. The addition of neoadjuvant and adjuvant radiochemotherapy allowed a further increase of the 5-year survival rate up to approximately $70 \%$.

Over the last 30 years laparoscopic surgery of rectal cancer has been progressively gaining popularity due to expanding experience and the development of technical skills according to the surgeons' learning curves [1]. Most of the available literature mentions many shortterm benefits of laparoscopic surgery over open surgery in rectal cancer treatment, such as less postoperative pain, decreased blood loss, and shorter duration of hospital stay. In terms of oncological outcomes (overall survival (OS), disease-free survival (DFS), local recurrence rate $(L R)$, distant recurrence rate (DR), number of harvested lymph nodes (LN), circumferential margin (CRM) positivity rate), in recent trials and meta-analyses it has been proven that laparoscopic surgery is not inferior to open surgery and can be safely and feasibly used $[2,3]$. Although available non-inferiority analyses suggest that the results of laparoscopic procedures are not worse than those of open surgery, the long-term results of this strategy remain debatable.
The following report constitutes a review of recently published articles concerning surgical techniques in colon cancer, with particular emphasis on rectal cancer. We address the question of whether, in view of further development and broader use of laparoscopic surgery, there is still a place for open surgery in the treatment strategy of rectal cancer.

\section{Risk of conversion}

Laparoscopic surgeries in colon cancer, including rectal cancer, are associated with significant risk of conversion to open technique, which varies from $1.5 \%$ up to $34 \%$, depending on the study [4]. Conditions requiring conversion to open surgery represent limitations of laparoscopy and are simultaneously procedures in which open surgery will continue to hold an advantage.

Like every other surgical technique, laparoscopy requires a certain degree of proficiency and is dependent on the operator's learning curve. Son et al. proved that 79 laparoscopic rectal resections are the break point, and the rate of complications as well as conversions decrease thereafter [5]. However, it should be underlined that laparoscopic surgeries and other advanced robot-assisted techniques should be utilised by surgeons with enough experience in open surgery to complete the procedure with adequate quality in case of conversion. For that reason, continuous training in open surgery should not be 
aborted because only open surgery warrants successful completion of the procedure after conversion.

According to Nienhüser et al., conditions that may be associated with risk of conversion to open surgery include: large tumour size, excessive tumour burden, and uncertainty about radical resection (which, in total, account for $17.7 \%$ of all conversion cases), as well as excessive intraabdominal adhesions, difficulties in splenic flexure release and limited access to the tumour (14.5\%), obesity (14.5\%), narrow pelvis (12.7\%), and uncertainty of the following: technical limitations associated with poor visibility, lack of expertise of the equipment or the equipment itself (9.1\%), bleeding (3.6\%), inability to perform safe resection of the distal part of the rectum (2.7\%), damage to the ureter (1.4\%), and other reasons (e.g. poor pulmonary condition, damage to adjacent organs) (9.1\%) [4]. Several of these aspects are examined below.

\section{The impact of conversion on surgical outcomes}

Kim et al. evaluated the effect of the conversion moment on the short-term and oncological effects of the surgery [6]. In their study, the term "early conversion" was used for a conversion decision that was made within 60 min and "late conversion" when it was made over $60 \mathrm{~min}$ from the beginning of the procedure. The most common conversion reasons reported by the authors were the presence of a PT4 tumour and positive history of previous abdominal surgeries. The mean operative time was longer in the late conversion. The rates of 30-day postoperative complications ( $42 \%$ vs. $27 \%$ ), Clavien-Dindo score $\geq 3$ (22\% vs. $11 \%)$, intensive care unit (ICU) care (31\% vs. 15\%), and transfusion (37\% vs. $21 \%)$ were significantly higher in the late conversion group. The authors concluded that decisions about early conversion made within 60 min of the beginning of surgery do not worsen short-term and oncologic outcomes.

In the MRC CLASICC Trial [7] study it was proven that, in patients who underwent conversion, the death rate was higher compared to patients operated by laparotomy and laparoscopy ( $9 \%$ vs. $5 \%$ vs. $1 \%$, respectively). Moreover, the following complications were more frequent in these patients: postoperative wound infection ( $20 \%$ vs. $12 \%$ vs. $10 \%$ ), upper respiratory tract infections (15\% vs. $5 \%$ vs. $8 \%$ ), anastomotic leak ( $15 \%$ vs. $7 \%$ vs. $8 \%$ ), and others ( $43 \%$ vs. $25 \%$ vs. $19 \%$ ). In the study an overall $34 \%$ conversion rate was observed. If the risk of conversion is high and conversion has a negative effect on the short-term outcomes of surgery, a laparoscopic approach is not recommended. Furthermore, in the CLASICC study, a significantly lower 5-year survival rate was observed in patients who required conversion [8].

Most of the available trials inspected the shortterm effect of the conversion on the surgery outcomes; however, there is a need for large studies assessing the effect of conversion long-term oncological treatment efficacy.

\section{Obesity and male patients}

In an article published in 2017 [9], Jayne et al. observed a significantly increased risk of conversion in obese patients compared to patients with underweight or normal body size (adjusted OR $=4.69,95 \% \mathrm{Cl}$ : $2.08-10.58 ; p<0.001)$. The second important observation was significantly more frequent conversions in men compared to women (adjusted OR $=2.44,95 \% \mathrm{Cl}$ : $1.05-5.71, p=0.04)$. As many as $16 \%$ of men required conversion to laparotomy, while in a group of women it was only $4.1 \%$. Similar observations often appear in the literature.

\section{Intra-abdominal adhesions}

Most operations in the abdominal cavity are associated with the appearance of intra-abdominal adhesions in the postoperative period.

Kim et al. [10] performed a study on patients undergoing colon cancer surgery with a positive history of a previous abdominal surgery. The authors concluded that intestinal manipulation during abdominal surgery induces postoperative adhesion formation. Intestinal manipulations are more often necessary during gastrointestinal surgery than in the case of operations not related to the gastrointestinal tract (e.g. gynaecological surgery), thus gastrointestinal operations more often cause adhesions that are more difficult to safely separate. The risk of conversion from the laparoscopic to open method was higher in the group of patients with positive history of previous gastrointestinal tract surgeries. However, the study concluded that laparoscopy may be the method of choice in patients with a positive history of surgery in the abdominal cavity but not related to the gastrointestinal tract.

\section{Locally advanced tumour (TNM c'T4)}

Because of the large size of the colon cancer cT4, and infiltration of surrounding tissues and adjacent organs, laparoscopic operations are extremely difficult and carry a high risk. Generally, in most of large studies, cases of T4 tumours were rare or not taken into account at all, hence the knowledge and research on laparoscopic resection are limited. Even if these patients are undergoing neoadjuvant treatment, downstaging is not always the case. 
In the work published by Yang et al. [11], patients with cT4 tumours were operated. In the group of patients operated on using the open method, in 21 cases, multivisceral resections were needed (the most frequently infiltrated organs were: small intestine, gynaecological organs, and duodenum). In contrast, in the group of patients undergoing laparoscopic surgery only five such cases were found, and the difference was statistically significant $(p=0.001)$. This is consistent with the results of available trials. Data published in study demonstrate that tumour stage cT4 should be an important consideration for surgeons when qualifying patients for open rather than laparoscopic surgery, because it is very difficult to achieve the goal of complete resection using the "no touch" principle (sharp separation, blood vessels first, tumour isolation, etc.). To reduce the risk of conversion and ensure the safety of surgery, the authors recommend qualification for laparoscopic surgery only in experienced centres, in patients with cT4 tumour size below $5 \mathrm{~cm}$ and with a maximum of one organ being infiltrated.

The American Joint Committee on Cancer (AJCC) classifies T4 colorectal cancers as those that invade into other organs and structures and/or perforate the visceral peritoneum. The AJCC and European Association of Endoscopic Surgery do not recommend laparoscopic treatment of all pathological T4 colorectal cancer [8].

Furthermore, Feinberg et al. emphasised in their meta-analysis that in clinical practice, due to the significant technical challenges, laparoscopic attempts of multivisceral resection should be reserved only to experts in advanced laparoscopic surgery [12].

\section{Technical aspects}

Laparoscopic instruments have their limitations. This is particularly important in the case of surgery of low rectal tumours, in patients with narrow and deep pelvis. Chand et al. [13] stressed in their study that such an operation may be exceptionally demanding when using rigid laparoscopic instruments with a limited range of motion. Development of tools that would allow free manoeuvring, especially in the narrow pelvis, and achieving the right angles during surgery is needed.

\section{Pathological outcomes}

The main goal of the TME procedure is total mesorectal excision and achievement of a negative circumferential resection margin (negative CRM). Meeting both of these criteria significantly reduces the risk of local recurrence of rectal cancer. In 2017 Martínez-Pérez et al. conducted a meta-analysis and systematic review evaluating pathological outcomes of laparoscopic vs. open mesorectal excision for rectal cancer [14]. The aim of the study was to evaluate the frequency of positive circumferential resection margin (CRM), defined as $1 \mathrm{~mm}$ or less from the closest tumour to the cut edge of the tissue, and the quality of mesorectal excision (complete, nearly complete, or incomplete). The study found that positive CRM was $17 \%$ more common in patients operated laparoscopically (7.9\%) than in those operated by open method (6.1\%), although this difference was not statistically significant. However, non-complete (nearly complete or incomplete) mesorectal excision was 31\% more frequent in patients in the laparoscopic group (13.2\%) than in the open group (10.4\%), and the difference was statistically significant. These reports question the oncological safety of laparoscopic surgery in the case of rectal cancer.

\section{Summary}

Open surgery should be considered as a first choice if the risk of conversion is high. Conversion may have an impact on early outcomes of surgery (increasing the risk of perioperative mortality and infectious complications) and long-term effects (worsening the prognosis).

Only continuous training in open techniques and routine conduction of these procedures will allow the high quality of the surgery to be maintained in case of conversion during laparoscopy.

Due to significantly more frequent non-complete mesorectal excision in the laparoscopic method, and thus the greater risk of local recurrence of cancer in the pelvis, further large randomised trials assessing homogeneous groups of patients with similar characteristics of rectal tumour are necessary.

\section{Conflict of interest}

The authors declare no conflict of interest.

\section{References}

1. Jacobs M, Verdeja JC, Goldstein HS. Minimally invasive colon resection (laparoscopic colectomy). Surg Laparosc Endosc 1991; 1: 144-50.

2. Zheng J, Feng X, Yang Z, et al. The comprehensive therapeutic effects of rectal surgery are better in laparoscopy: a systematic review and meta-analysis. Oncotarget 2017; 8: 12717-29.

3. Chen K, Cao G, Chen B, et al. Laparoscopic versus open surgery for rectal cancer: a meta-analysis of classic randomized controlled trials and high-quality nonrandomized studies in the last 5 years. Int J Surg 2017; 39: 1-10.

4. Nienhüser $\mathrm{H}$, Heger P, Schmitz R, et al. Short- and long-term oncological outcome after rectal cancer surgery: a systematic review and meta-analysis comparing open versus laparoscopic rectal cancer surgery. J Gastrointest Surg 2018; 22: 1418-27.

5. Son GM, Kim JG, Lee JC, et al. Multidimensional analysis of the learning curve for laparoscopic rectal cancer surgery. J Laparoendosc Adv Surg Tech 2010; 20: 609-17. 
6. Kim IY, Kim BR, Kim YW. Impact of timing of conversion to open surgery on short-term and oncologic outcomes in patients undergoing minimally invasive surgery for colorectal cancer. Am Surg 2017; 83: 71-7.

7. Guillou PJ, Quirke P, Thorpe H, et al. Short-term endpoints of conventional versus laparoscopic-assisted surgery in patients with colorectal cancer (MRC CLASICC trial): multicentre, randomised controlled trial. Lancet 2005; 365: 1718-26.

8. Mathis KL, Nelson H. Controversies in laparoscopy for colon and rectal cancer. Surg Oncol Clin N Am 2014; 23: 35-47.

9. Jayne D, Pigazzi A, Marshall H, et al. Effect of robotic-assisted vs conventional laparoscopic surgery on risk of conversion to open laparotomy among patients undergoing resection for rectal cancer the ROLARR randomized clinical trial. JAMA 2017 318: 1569-80.

10. Kim YW, Kim IY. Comparison of the short-term outcomes of laparoscopic and open resections for colorectal cancer in patients with a history of prior median laparotomy. Indian J Surg 2017; 79: 527-33.

11. Yang ZF, Wu DQ, Wang JJ, et al. Short- and long-term outcomes following laparoscopic vs open surgery for pathological T4 colorectal cancer: 10 years of experience in a single center. World J Gastroenterol 2018; 24: 76-86.

12. Feinberg AE, Chesney TR, Acuna SA, et al. Oncologic outcomes following laparoscopic versus open resection of pT4 colon cancer: a systematic review and meta-analysis. Dis Colon Rectum 2017; 60: 116-25.

13. Chand M, Moran B, Wexner SD. Which technique to choose in the high-tech era of minimal-access rectal cancer surgery? Colorectal Dis 2016; 18: 839-41.

14. Martínez-Pérez A, Carra MC, Brunetti F, De’Angelis N. Pathologic outcomes of laparoscopic vs open mesorectal excision for rectal cancer: a systematic review and meta-analysis. JAMA 2017; 152: e165665.

Received: 18.08 .2018

Accepted: 13.09 .2018 